\title{
THE ALGEBRAIC CLOSURE IN FUNCTION FIELDS OF QUADRATIC FORMS IN CHARACTERISTIC 2
}

\author{
Hamza Ahmad
}

\begin{abstract}
For a field $k$ of characteristic not two, it is known that $k$ is algebraically closed in the function field of any (non-degenerate) quadratic form in three or more variables. In this note we consider fields of characteristic two and decide when $k$ is algebraically closed in a function field of a quadratic $k$-form. For quadratic forms in three variables this has recently been done by Ohm.
\end{abstract}

\section{INTRODUCTION}

Let $k$ be a field of characteistic not two. Then $k$ is algebraically closed in the function field of any (non-degenerate) quadratic $k$-form in three or more variables. This is because such forms are absolutely irreducible (that is, they remain irreducible over the algebraic closure of $k$ ) and therefore their function fields are regular (see [3, p.18, Theorem 5]).

In this note we take $k$ to be a field of characteristic two, and $Q$ to be an irreducible quadratic $k$-form. We answer the following.

QUESTION: When is $k$ algebraically closed in the function field of $Q$ ? For function fields of conics, the question has been answered by Ohm in [2, 2.8-2.12].

Terminology and Preliminaries: By a quadratic $k$-form $Q(X)$ we mean a homogeneous polynomial of degree 2 in the variables $X=\left(X_{1}, \ldots, X_{n}\right)$ with coefficients from $k$. If $Q$ is irreducible, then by the function field $k(Q)$ of $Q$ over $k$ we mean the field of fractions of the integral domain $k[X] /(Q)$, where $(Q)$ denotes the ideal in $k[X]$ generated by the polynomial $Q$. Therefore an extension $K / k$ is (isomorphic to) the function field of $Q\left(X_{0}, \ldots, X_{n}\right)$ if and only if $K=k\left(x_{0}, \ldots, x_{n}\right)$ such that $Q(x)=0$ and the transcendence degree of $K / k$, abbreviated $\operatorname{dt}(K / k)$, equals $n$. If $Q^{\prime}$ is obtained from $Q$ by means of an invertible linear change of variables, then $k(Q)$ and $k\left(Q^{\prime}\right)$ are $k$-isomorphic.

Received 18th April, 1996.

The author wishes to thank Professor Ohm for his encouragement to work on the question and for many valuable discussions.

Copyright Clearance Centre, Inc. Serial-fee code: 0004-9729/97 \$A2.00+0.00. 
Over a field $k$ of characteristic two, any quadratic $k$-form can be written (after an invertible linear change of variables) as

$$
Q(X, Y, Z)=\sum_{i=1}^{r}\left(a_{i} X_{i}^{2}+X_{i} Y_{i}+b_{i} Y_{i}^{2}\right)+\sum_{i=1}^{\infty} c_{i} Z_{i}^{2}
$$

where $a_{i}, b_{i}, c_{i} \in k$ and $r, s \geqslant 0$ (see [1]).

\section{The Results}

REMARK 1. Let $Q$ be as in (*) above. Direct calculation shows that

(1) $Q$ is reducible if and only if either

$$
\begin{aligned}
& \text { i. } r=0 \text { and } c_{i} / c_{j} \in k^{2} \text { for } 1 \leqslant i, j \leqslant s \text { and } c_{j} \neq 0 \text {, or } \\
& \text { ii. } s=0 \text { and } r=1 \text { and } a_{1} T^{2}+T+b_{1} \text { is reducible in } k[T] \text {. }
\end{aligned}
$$

(2) $Q$ is absolutely irreducible if and only if either $r \geqslant 2$, or $r=1$ and $c_{i} \neq 0$ for some $i$.

If $Q$ is absolutely irreducible, then its function field is regular and therefore $k$ is algebraically closed in $k(Q)$. If $r=1$ and $c_{i}=0$ for $1 \leqslant i \leqslant s$, then $Q=$ $a_{1} X^{2}+X Y+b_{1} Y^{2}$ and therefore $k$ is not algebraically closed in $k(Q)$. It remains to discuss the question when $r=0$; that is, when $Q$ is diagonal. This is done in our theorem below.

REMARK 2. Let $k$ be a field of characteristic 2 , and $a_{1}, \ldots, a_{n} \in k$. Let $z_{1}, \ldots, z_{n}$ be algebraically independent elements over $k$. Then the polynomial

$$
Z^{2}+\left(a_{1} z_{1}^{2}+\cdots+a_{n} z_{n}^{2}\right)
$$

in one variable $Z$ over $L:=k\left(z_{1}, \ldots, z_{n}\right)$ is reducible if and only if $\sqrt{a_{i}} \in k$ for all $1 \leqslant i \leqslant n$.

PROOF: The $L$-polynomial $Z^{2}+\left(a_{1} z_{1}^{2}+\cdots+a_{n} z_{n}^{2}\right)$ is reducible over $L$ if and only if $a_{1} z_{1}^{2}+\cdots+a_{n} z_{n}^{2}$ is a square in $L$. That is, $\left(a_{1} z_{1}^{2}+\cdots+a_{n} z_{n}^{2}\right) g^{2}=f^{2}$ where $f$ and $g$ and $k$-polynomials in (the algebraically independent elements) $z_{1}, \ldots, z_{n}$. Comparing the leading coefficients of $z_{i}$ in the last equation, we have $a_{i} \in k^{2}$.

The following lemma will serve as the inductive step for the proof of our theorem and is due to $\mathrm{Ohm}$ (see $[2,2.12]$ ).

LEMMA. Let $k$ be a field of characteristic 2 and let $Q(X, Y, Z)=X^{2}+a Y^{2}+$ $b Z^{2}$ be an irreducible $k$-form such that $k$ is not algebraically closed in $k(Q)$. Then $[k(\sqrt{a}, \sqrt{b}): k]=2$.

Proof: The function field $k(Q)$ equals $k(y, z)\left(\sqrt{\left(a y^{2}+b z^{2}\right)}\right)$ with $y, z$ algebraically independent over $k$. Set $\alpha=\sqrt{\left(a y^{2}+b z^{2}\right)}$. By hypothesis there exists 
$d \in k(Q)$ algebraic over $k$ and $d \notin k$. Since $k(y, z)$ is pure transcendental over $k, d \notin k(y, z)$. Therefore $k(Q)=k(y, z)(\alpha)=k(d)(y, z)$. In particular, $[k(d): k]=2$. Also, the polynomial $X^{2}+\left(a y^{2}+b z^{2}\right)$ is reducible over $k(d)(y, z)$. By Remark 2, we have $\sqrt{a}, \sqrt{b} \in k(d)$; hence $[k(\sqrt{a}, \sqrt{b}): k]=[k(d): k]=2$.

ThEOREM. Let $k$ be a field of characteristic 2 and let $Q(X)=X_{0}^{2}+a_{1} X_{1}^{2}+\cdots+$ $a_{n} X_{n}^{2}$ be an irreducible quadratic $k$-form. Then $k$ is algebraically closed in $k(Q)$ if and only if $\left[k\left(\sqrt{a_{1}}, \sqrt{a_{2}}, \ldots, \sqrt{a_{n}}\right): k\right] \geqslant 4$.

Proof: As in the introduction, $k(Q)=k\left(x_{0}, \ldots, x_{n}\right)$ such that

$$
x_{0}^{2}+a_{1} x_{1}^{2}+\cdots+a_{n} x_{n}^{2}=0
$$

and the transcendence degree of $k\left(x_{0}, \ldots, x_{n}\right) / k$ equals $n$. For the rest of the proof let $L:=k\left(\sqrt{a_{1}}, \sqrt{a_{2}}, \ldots, \sqrt{a_{n}}\right)$.

For $n=1$, the $k$-polynomial $T^{2}+a_{1}$ has a root in $k(Q)$, hence the theorem is true. Now, let $n>1$. Assume first that $[L: k]=2$. By symmetry, we may assume that $\sqrt{a_{1}} \notin k$, and for $i>1, \sqrt{a_{i}}=\alpha_{i}+\beta_{i} \sqrt{a_{1}}$ where $\alpha_{i}, \beta_{i} \in k$. Substituting in (1) we get

$0=\left(x_{0}+\alpha_{2} x_{2}+\cdots+\alpha_{n-1} x_{n-1}+\alpha_{n} x_{n}\right)+\sqrt{a_{1}}\left(x_{1}+\beta_{2} x_{2}+\cdots+\beta_{n-1} x_{n-1}+\beta_{n} x_{n}\right)$.

If $x_{1}+\beta_{2} x_{2}+\beta_{3} x_{3}+\cdots+\beta_{n} x_{n}=0$, then $x_{0}+a_{2} x_{2}+\alpha_{3} x_{3}+\cdots+\alpha_{n} x_{n}=0$ and therefore $x_{1}, x_{2} \in k\left(x_{2}, \ldots, x_{n}\right)$. Hence $\operatorname{dt}(k(Q) / k) \leqslant n-1 ;$ a contradiction. So, $x_{1}+\beta_{2} x_{2}+\beta_{3} x_{3}+\cdots+\beta_{n} x_{n} \neq 0$. Then the last displayed equation implies that

$$
\sqrt{a_{1}}=\frac{x_{0}+\alpha_{2} x_{2}+\cdots+\alpha_{n-1} x_{n-1}+\alpha_{n} x_{n}}{x_{1}+\beta_{2} x_{2}+\cdots+\beta_{n-1} x_{n-1}+\beta_{n} x_{n}} \in k(Q) \text {. }
$$

Thus $\sqrt{a_{1}} \in k(Q)$ and $\sqrt{a_{1}} \notin k$. Therefore $k$ is not algebraically closed in $k(Q)$.

Now assume that $[L: k] \geqslant 4$. We want to show that in this case $k$ is algebraically closed in $k(Q)$. We use induction on $n$. The case $n=2$ follows from the Lemma above. So assume that $n>2$.

We first treat the case $[L: k]=4$. Without loss of generality, we may assume that $L=k\left(\sqrt{a_{1}}, \sqrt{a_{2}}\right)$. Since $n>2$, we have $\sqrt{a_{n}} \in k\left(\sqrt{a_{1}}, \sqrt{a_{2}}\right)$. Therefore $\sqrt{a_{n}}=\alpha+\beta \sqrt{a_{1}}+\gamma \sqrt{a_{2}}+\delta \sqrt{a_{1} a_{2}}$, which implies that $a_{n}=\alpha^{2}+\beta^{2} a_{1}+\gamma^{2} a_{2}+\delta^{2} a_{1} a_{2}$ where $\alpha, \beta, \gamma, \delta \in k$. Substituting in (1), we get

$$
\begin{aligned}
0= & \left(x_{0}+\alpha x_{n}\right)^{2}+a_{1}\left(x_{1}+\beta x_{n}\right)^{2}+a_{2}\left(x_{2}+\gamma x_{n}\right)^{2}+a_{3} x_{3}^{2} \\
& \quad+\cdots+a_{n-1} x^{2}+a_{1} a_{2} \delta^{2} x_{n}^{2} \\
= & y_{0}^{2}+a_{1} y_{1}^{2}+a_{2} y_{2}^{2}+a_{3} y_{3}^{2}+\cdots+a_{n-1} y_{n-1}^{2}+a_{1} a_{2} \delta^{2} y_{n}^{2}
\end{aligned}
$$


where $y_{0}=x_{0}+\alpha x_{n}, y_{1}=x_{1}+\beta x_{n}, y_{2}=x_{2}+\gamma x_{n}$, and $y_{i}=x_{i}, i \geqslant 3$. Note that $k(Q)=k\left(x_{0}, \ldots, x_{n}\right)=k\left(y_{0}, \ldots, y_{n}\right)$. In particular, the transcendence degree of the field $K:=k\left(y_{0}, \ldots, y_{n-1}\right) / k \geqslant n-1$.

If $\delta=0$, then from equation (2) we conclude that $\operatorname{dt}(K / k)=n-1$ and that $K$ is the function field of the quadratic form $Y_{0}^{2}+a_{1} Y_{1}^{2}+a_{2} Y_{2}^{2}+a_{3} Y_{3}^{2}+$ $\cdots+a_{n-1} Y_{n-1}^{2}$. Therefore by the inductive hypothesis, $k$ is algebraically closed in $K$ since $\left[k\left(\sqrt{a_{1}}, \sqrt{a_{2}}, \cdots, \sqrt{a_{n-1}}\right): k\right]=4$. Now since $k(Q)=K\left(y_{n}\right)$ and $\operatorname{dt}(k(Q) / k)=1+d t(K / k), y_{n}$ is transcentdental over $K$. Therefore $k$ is algebraically closed in $K\left(y_{n}\right)=k(Q)$.

On the other hand, if $\delta \neq 0$, then from (2) we have

$$
0=y_{0}^{2}+a_{1} y_{1}^{2}+a_{2}\left(y_{2}^{2}+a_{1} \delta^{2} y_{n}^{2}\right)+a_{3} y_{3}^{2}+\cdots+a_{n-1} y_{n-1}^{2} .
$$

If $y_{2}^{2}+a_{1} \delta^{2} y_{n}^{2}=0$, then $y_{2}$ is algebraic over $k\left(y_{n}\right)$, and equation (3) implies that $y_{0}$ is algebraic over $k\left(y_{1}, \ldots, y_{n-1}\right)$. Hence $y_{0}$ and $y_{2}$ are algebraic over $k\left(y_{1}, y_{3}, \ldots, y_{n-1}\right)$. This implies that $\mathrm{dt}\left(k\left(y_{0}, \ldots, y_{n}\right) / k\right) \leqslant n-1$. But $k(Q)=k\left(y_{0}, \ldots, y_{n}\right)$ has transcendence degree $n$, a contradiction. Therefore $y_{2}^{2}+a_{1} \delta^{2} y_{n}^{2} \neq 0$. Now by setting $z_{n}=\delta y_{n} / y_{2}, z_{0}=\left(y_{0}+a_{1} y_{1} z_{n}\right) /\left(1+a_{1} z_{n}^{2}\right), z_{1}=\left(y_{1}+y_{0} z_{n}\right) /\left(1+a_{1} z_{n}^{2}\right), z_{2}=y_{2}$, and for $3 \leqslant i<n, z_{i}=y_{i}$ and $A_{i}=a_{i} /\left(1+a_{1} z_{n}^{2}\right) \in k\left(z_{n}\right)$, we have from equation (3)

$$
0=\left(1+a_{1} z_{n}^{2}\right)\left(z_{0}^{2}+a_{1} z_{1}^{2}+a_{2} z_{2}^{2}+A_{3} z_{3}^{2}+\cdots+A_{n-1} z_{n-1}^{2}\right),
$$

and therefore

$$
0=z_{0}^{2}+a_{1} z_{1}^{2}+a_{2} z_{2}^{2}+A_{3} z_{3}^{2}+\cdots+A_{n-1} z_{n-1}^{2} .
$$

Also note that $k(Q)=k\left(z_{n}\right)\left(z_{0}, \ldots, z_{n-1}\right)$ and the transcendence degree of $k(Q) / k\left(z_{n}\right)$ equals $n-1$. Therefore $k(Q) / k\left(z_{n}\right)$ is the function field of the $k\left(z_{n}\right)$-quadratic form

$$
Z_{0}^{2}+a_{1} Z_{1}^{2}+a_{2} Z_{2}^{2}+A_{3} Z_{3}^{2}+\cdots+A_{n-1} Z_{n-1}^{2} \text {. }
$$

Since $\left[k\left(z_{n}\right)\left(\sqrt{a_{1}}, \sqrt{a_{2}}, \sqrt{A_{3}}, \ldots, \sqrt{A_{n-1}}\right): k\left(z_{n}\right)\right] \geqslant 4$, the inductive hypothesis implies that $k\left(z_{n}\right)$ is algebraically closed in $k\left(z_{n}\right)\left(z_{0}, \ldots, z_{n-1}\right)=k(Q)$. In particular, $k$ is algebraically closed in $k(Q)$. This concludes the case $[L: k]=4$.

To finish the proof of the theorem, it is left to show that $K$ is algebraically closed in $k(Q)$ when $[L: k] \geqslant 8$. In this case we may assume, without loss of generality, that $\sqrt{a_{2}} \notin k\left(\sqrt{a_{1}}\right) \neq k$. Let $x=x_{0}-\sqrt{a_{1}} x_{1} \in k(Q)\left(\sqrt{a_{1}}\right)$. Then from equation (1) we have

$$
0=x^{2}+a_{2} x_{2}^{2}+\cdots+a_{n} x_{n}^{2},
$$

and $\operatorname{dt}\left(k\left(\sqrt{a_{1}}\right)\left(x, x_{2}, \ldots, x_{n}\right) / k\left(\sqrt{a_{1}}\right)\right)=n-1$. Thus $k\left(\sqrt{a_{1}}\right)\left(x, x_{2}, \ldots, x_{n}\right)$ is the function field of the $k\left(\sqrt{a_{1}}\right)$-form $X^{2}+a_{2} X_{2}^{2}+\cdots+a_{n} X_{n}^{2}$. By the inductive hypothesis, $k\left(\sqrt{a_{1}}\right)$ is algebraically closed in $k\left(\sqrt{a_{1}}\right)\left(x, x_{2}, \ldots, x_{n}\right)$ because 
$\left[k\left(\sqrt{a_{1}}, \sqrt{a_{2}}, \ldots, \sqrt{a_{n}}\right): k\left(\sqrt{a_{1}}\right)\right] \geqslant 4$. Since $k\left(\sqrt{a_{1}}\right)\left(x, x_{2}, \ldots, x_{n}\right)\left(x_{1}\right)=k\left(\sqrt{a_{1}}\right)$ $\left(x_{0}, \ldots, x_{n}\right)$ and $x_{1}$ is transcendental over $k\left(\sqrt{a_{1}}\right)\left(x, x_{2}, \ldots, x_{n}\right)$, it follows that $k\left(\sqrt{a_{1}}\right)$ is algebraically closed in $k\left(\sqrt{a_{1}}\right)\left(x_{0}, \ldots, x_{n}\right)$. By symmetry, $k\left(\sqrt{a_{2}}\right)$ is also algebraically closed in $k\left(\sqrt{a_{2}}\right)\left(x_{0}, \ldots, x_{n}\right)$. Now if $d \in k\left(x_{0}, \ldots, x_{n}\right)$ is algebraic over $k$ (and thus algebraic over $k\left(\sqrt{a_{1}}\right)$ and $k\left(\sqrt{a_{2}}\right)$ ), then $d \in k\left(\sqrt{a_{1}}\right)$ and $d \in k\left(\sqrt{a_{2}}\right)$. Thus $d \in k$. Thus $k$ is algebraically closed in $k(Q)$.

\section{REFERENGES}

[1] C. Arf, 'Untersuchungen über quadratische Formen in Körpern der Charakteristik 2', J. Reine Angew Math. 183 (1941), 148-167.

[2] J. Ohm, 'Function fields of conics, a theorem of Amitsur-MacRae, and a problem of Zariski', in Algebraic geometry and its applications, (C. Bajaj, Editor) (Springer-Verlag, Berlin, Heidelberg, New York, 1994), pp. 333-363.

[3] A. Weil, Foundations of algebraic geometry, Amer. Math. Soc. Colloq. Publ. 29 (American Mathematical Society, Providence, RI, 1962).

Department of Mathematics

Yarmouk University

Irbid

Jordan 